\title{
ANÁLISIS DE LAS NUEVAS PRERROGATIVAS DEL AUTOR SOBRE LAS OBRAS DERIVADAS DE LA SUYA -A LA LUZ DEL ARTÍCULO 21 DEL TRLPI EN ESPAÑA*
}

\author{
ANALYSIS OF THE NEW AUTHORS' \\ PREROGATIVES FROM HIS/HER \\ DERIVATIVE WORKS - IN LIGHT OF \\ ARTICLE 21 OF TRLPI IN SPAIN
}

\author{
Juan Camilo Contreras-Jaramillo**
}

Fecha de recepción: 16 de febrero de 2014 Fecha de aceptación: 30 de febrero de 2014

Disponible en linea: 30 de julio de 2014

\section{Para citar este artículo/To cite this article}

\begin{abstract}
Contreras-Jaramillo, Juan Camilo, Análisis de las nuevas prerrogativas del autor sobre las obras derivadas de la suya - a la luz del artículo 21 del TRLPI en España, 129 Vniversitas, 81-102 (2014). http://dx.doi. org/10.11144/Javeriana.VJ129.anpa

doi:10.11144/Javeriana.VJ129.anpa
\end{abstract}

Artículo realizado en desarrollo del proyecto Derecho y Propiedad, del grupo de investigación en derecho privado de la Facultad de Ciencias Jurídicas, Pontificia Universidad Javeriana.

** Profesor del Departamento de Derecho Privado en la Facultad de Ciencias Jurídicas, Pontificia Universidad Javeriana. Abogado de la misma Universidad. Especialista en propiedad industrial, derecho de autor y nuevas tecnologías, Universidad Externado de Colombia. Máster en propiedad intelectual, Universidad Carlos III de Madrid y candidato a doctor, Pontificia Universidad Javeriana. Correo electrónico: juancontreras@javeriana.edu.co. 


\section{RESUMEN}

El artículo 21 del Real Decreto Legislativo 1/1996, de 12 de abril, del Texto Refundido de la Ley de Propiedad Intelectual, TRLPI, no solo consagra el derecho de transformación del autor sobre su propia obra, además, abre la posibilidad a la participación del mismo autor como titular de derechos de propiedad intelectual sobre las obras derivadas que sean producidas por terceros con su autorización; esto es, conservando la potestad para autorizar cualquier uso de la obra resultante. Esta reinterpretación de lo que la doctrina ha entendido por el derecho de transformación es la consecuencia no querida de la implementación de una Directiva Europea sobre bases de datos. Este artículo de reflexión sobre la disposición anteriormente citada pretende hacer evidente el ensanchamiento de las facultades de los titulares de derechos sobre las obras derivadas en la legislación española.

Palabras clave: propiedad intelectual; derecho de autor; obras derivadas; derecho de transformación; texto refundido de la ley de propiedad intelectual; obras colectivas; obras en colaboración; bases de datos. 


\section{ABSTRACT}

Article 21 of the Spanish Intellectual Property Law, Real Decreto Legislativo 1/1996, April 12, does not only established the derivative right of the protected works, but it enables the participation of the original author as a right holder of the derivative works made by another author with his consent; by giving a privilege to authorize any further use of the resultant work. Such reinterpretation of what the civil law doctrine, regarding intellectual property, has understood as the derivative right, comes from the implementation of the European Directive of protected databases. This article pretends to show the broadening rights of the right holders over derivative works in the Spanish law.

Key words: Intellectual property; copyright; derivative works; derivative right; Spanish intellectual property law; databases.

\section{SUMARIO}

INTRODUCCIÓN.- I. LAS OBRAS DERIVADAS EN LA PROPIEDAD INTELECTUAL.- II. LA REDACCIÓN DEL ARTÍCULO 21.2 DEL TRLPI Y SU RELECTURA.- III. ANTECEDENTES NORMATIVOS DE LA NUEVA TRANSFORMACIÓN.- IV. EL DROIT DE SUITE COMO EL AMBIENTE PROPICIO PARA EL SEGUIMIENTO DEL DERECHO DE AUTOR EN LAS OBRAS DERIVADAS.- CONCLUSIONES.- BibLIOGRAFía. 


\section{INTRODUCCIÓN}

En España, la Ley 21/2014, de 4 de noviembre, por la que se modifica el Texto Refundido de la Ley de Propiedad Intelectual, aprobado por Real Decreto Legislativo 1/1996, de 12 de abril, y la Ley 1/2000, de 7 de enero, de Enjuiciamiento Civil, es ya una realidad que entra en vigencia el 1 de enero de 2015; una realidad en la que seguramente habrá más polémica que consenso y más dudas que certezas. Temas como la copia privada, las facultades de la Comisión de Propiedad Intelectual respecto de usos infractores en internet, el nuevo canon compensatorio por la agregación de noticias, y la regulación de las entidades de gestión y de las obras huérfanas son los nuevos temas que esta reforma pone sobre la mesa, pero que no deberían desvanecer otras preguntas que aún flotan en el caótico ambiente de la propiedad intelectual.

Una de esas preguntas, que está estrechamente ligada con los nuevos temas de discusión, es la de la necesidad y los riesgos de una regulación detallada de todos los aspectos de la propiedad intelectual. Consecuencia de la antiquísima discusión sobre si el derecho debe interpretar la realidad al momento de la regulación o si, más bien, el legislador ostenta un deber de modulación o transformación de la misma realidad por medio de las leyes que emite.

Y allí, en el marco de esa discusión, la actual regulación del derecho de transformación y las obras derivadas recobra importancia. El artículo 21 del Texto Refundido de la Ley de Propiedad Intelectual ${ }^{1}$, producto de la implementación de una Directiva Europea en el derecho interno español, es muestra fehaciente de los riesgos que una "superregulación" puede generar en el sistema de la propiedad intelectual, cuando su objetivo es adecuar la realidad a un propósito político; en este caso, implementar a cualquier costo una Directiva Europea sobre bases de datos.

El resultado, el artículo 21 de la Ley de Propiedad Intelectual española, como veremos, termina otorgando una ampliación desmedida de los derechos del autor original sobre las obras deriva-

1 España, Real Decreto Legislativo 1/1996, de 12 de abril, por el que se aprueba el texto refundido de la Ley de Propiedad Intelectual, regularizando, aclarando y armonizando las disposiciones legales vigentes sobre la materia, 97 Boletín Oficial del Estado, BOE, 22 de abril de 1996. Disponible en: https://www.boe.es/diario_boe/txt.php?id=BOE-A-1996-8930 
das de la suya. Este problema retumba en el corazón del proceso creativo en relación con la "sobrescritura" o reinterpretación que los creadores han manejado siempre, construyendo bien sobre el dominio público, bien sobre obras protegidas, pero siempre, como se dice coloquialmente "parándose sobre los hombros de gigantes".

\section{LAS OBRAS DERIVADAS EN LA PROPIEDAD INTELECTUAL}

La facultad de transformación existe como derecho patrimonial o de explotación, con el fin de garantizar los diferentes modelos de negocio que sobre una misma obra pueden darse; pero a la vez para asegurar el tráfico económico al que ingresará esa misma obra ${ }^{2}$. De no ser así, la mayoría de las obras que llamamos derivadas ${ }^{3}$ sería inviable como objeto de comercio ${ }^{4}$. No es difícil observar que cualquiera de las obras enumeradas como derivadas en el artículo 11 del TRLPI tiene la altísima probabilidad de ser considerada como una modificación que vulnera la integridad de la obra original de la que proviene, en los términos del artículo 14 de la misma ley, es decir, un acto cercano al derecho irrenunciable, inalienable e imprescriptible (el de proteger la integridad de la obra) que tiene el autor como una facultad moral sobre su creación; esto sin más, dejaría las traducciones, adaptaciones, arreglos musicales y todo tipo de transformaciones en una situación de absoluta inseguridad jurídica, imposible de asumir por el mercado de la propiedad intelectual.

2 Tal como afirman William M. Landes \& Richard A. Posner, al otorgar un derecho de transformación al autor original, el cual puede ceder o licenciar, se están reduciendo los costos de transacción en la medida en que el usuario de la obra derivada deberá negociar solo con un titular de derechos, no con dos. William M. Landes \& Richard A. Posner, An Economic Analysis of Copyright Law, 18 Journal of Legal Studies, 2, 325-363, 325-333, 344-353 (1989). Disponible en: http://www.sfu.ca/ allen/copyright.pdf

3 Más allá de las diferencias doctrinales sobre si las obras compuestas constituyen una categoría que subsume a las derivadas, a efectos del presente artículo, entendemos obra derivada como sinónimo de obra compuesta, como lo han interpretado la doctrina francesa o la española. Hermenegildo Baylos-Corroza, Tratado de Derecho Industrial, 789, 3ª ed., Civitas, Navarra (2009).

4 Sobre otras críticas a las obras derivadas como infracciones al derecho del autor original, Glynn S. Lunney, JR., Copyright, Derivative Works, and the Economics of Complements, 12 Vanderbilt Journal of Entertainment and Technology Law, 4, 779-817 (2010). Disponible en: http://www.jetlaw.org/wp-content/uploads/2011/09/Lunney_online.pdf 
De allí que las que llamamos obras derivadas no se cataloguen como modificaciones sujetas al derecho moral 5 , sino que se encuadren como explotaciones patrimoniales de la obra, resultado del ejercicio del derecho de transformación ${ }^{6}$ (artículo 21 TRLPI) 7. Distinción simple pero necesaria al momento de llevar a cabo transacciones comerciales con las mencionadas obras, ya que los empresarios que las explotarán buscarán el ejercicio de su actividad económica, para garantizar el uso pacífico de estas obras y que, en consecuencia, no podrán estar sujetas al concepto subjetivo de integridad que tengan el autor o sus causahabientes mortis causa.

Por otro lado, a fin de identificar la titularidad de los derechos, al autor de la obra resultante de la transformación, más allá de si es derivada o compuesta, siempre se le han reconocido las dos facultades de la propiedad intelectual (morales y patrimoniales), ya que es él quien lleva a cabo la actividad creadora; ${ }^{8}$ la cual, por sí misma ${ }^{9}$ genera derechos sobre una obra que entrará, si así lo desea su autor, en el circuito comercial. Claro está que en el caso de las obras derivadas, el autor transformador deberá contar, primero, con la debida autorización del autor original para realizar tal transformación —o acto creador sobre una obra preexistente $-{ }^{10}$.

$\mathrm{Y}$ en cualquier caso, el producto de la transformación (obra derivada o compuesta), si tiene la característica de "original", constituirá un bien de propiedad intelectual complejo que integra una obra preexistente y una creación sobre la que se generan derechos de autor ex novo; este bien evidencia una "relación de filiación entre una obra y otra"11. Esta relación entre obra original y derivada

5 Rodrigo Bercovitz Rodríguez-Cano, coord., autor, Manual de propiedad intelectual, 95, Tirant Lo Blanch, Valencia (2001).

6 Derecho de transformación que, como a todos los de explotación, se le aplicarán todos los límites, la temporalidad y los condicionamientos legalmente establecidos en el TRLPI.

7 Cristina López-SÁnchez, La transformación de la obra intelectual, 66, Dykinson, Madrid (2008).

8 Para ampliar sobre el proceso de la actividad creadora, Jorge OrTEga-Doménech, Creación humana y autoría intelectual: diálogos en torno a su fundamentación jurídica, en Sujetos del derecho de autor, 13-42, 16 y ss. César Iglesias-Rebollo, coord., Reus, Madrid (2007).

9 "La propiedad intelectual de una obra literaria, artística o científica corresponde al autor por el solo hecho de su creación", artículo 1 TRLPI.

10 Pamela Samuelson, The Quest for a Sound Conception of Copyright's Derivate Work Right, 101 Georgetown Law Journal, 1505-1564 (2013). Disponible en: http://georgetownlawjournal. org/files/2013/09/Samuelson.pdf

11 Cristina López-Sánchez, La transformación de la obra intelectual, 66, Dykinson, Madrid (2008). 
siempre se ha entendido como la protección de la nueva creación "sin perjuicio" de los derechos del autor de la obra original, es decir, para incorporar la obra preexistente a la nueva creación se requiere la autorización del titular de derechos de la primera ${ }^{12}$.

\section{LA REDACCIÓN DEL ARTÍCULO 21.2 DEL TRLPI Y SU RELECTURA}

Pues bien, al leer cuidadosamente el artículo 21.2 del Texto Refundido de la Ley de Propiedad Intelectual, encontramos que esa seguridad jurídica que se busca en las facultades de explotación ${ }^{13}$ ha sido alterada, al ampliar los derechos exclusivos del autor de la obra preexistente hasta los resultados de la transformación que un tercero autorizado pueda hacer de su obra original.

El artículo 21.2 del TRLPI al determinar los parámetros del derecho de transformación establece:

Los derechos de propiedad intelectual de la obra resultado de la transformación corresponderán al autor de esta última, sin perjuicio del derecho del autor de la obra preexistente de autorizar, durante todo el plazo de protección de sus derechos sobre esta, la explotación de esos resultados en cualquier forma y en especial mediante su reproducción, distribución, comunicación pública o nueva transformación ${ }^{14}$.

Las palabras resaltadas ubican justamente esa ampliación de facultades del autor de una obra ${ }^{15}$, toda vez que ya no se limitan a la

12 Delia Lipszyc, Derecho de autor y derechos conexos, 112, 2 a ed., United Nations Educational, Scientific and Cultural Organization, UNESCO, Buenos Aires (1993).

13 La seguridad jurídica a la que se hace referencia, no solo trata sobre la distinción entre derechos morales y derechos de explotación, sino que también implica la unidad de criterio para determinar la autoría de una obra. Edward J. DAMICH, The Right of Personality: A Common-Law Basis for the Protection of the Moral Rights of Authors, 23 Georgia Law Review, 1-24 (1988).

14 España, Real Decreto Legislativo 1/1996, de 12 de abril, por el que se aprueba el texto refundido de la Ley de Propiedad Intelectual, regularizando, aclarando y armonizando las disposiciones legales vigentes sobre la materia, 97 Boletín Oficial del Estado, BOE, 22 de abril de 1996. Disponible en: https://www.boe.es/diario_boe/txt.php?id=BOE-A-1996-8930

15 Entendiendo la facultad de autorizar como ese derecho de explotación otorgado de forma exclusiva al titular. "Es un derecho 'exclusivo', de modo que solamente el autor - o su derechohabiente - puede realizar, autorizar o prohibir todo acto que implique el uso de su obra y ese derecho es oponible 'erga omnes', incluso frente al adquirente del soporte material que contiene la creación, a menos que una norma expresa o una cláusula contractual disponga lo contrario". RiCARDo ANTEQUERA, Las limitaciones y excepciones al derecho de autor y los derechos conexos en el entorno digital. XI Curso académico regional OMPI/SGAE sobre derecho de autor y derechos conexos para países de América Latina: el derecho de autor y los derechos 
simple autorización del autor original para incluir su obra en otra derivada, sino que, además, le permite a ese autor mantener algún grado de disposición respecto de la explotación de esos resultados (entiéndase, la obra derivada).

Un precepto básico de la propiedad intelectual es aquel en virtud del cual el autor de una obra original tiene el derecho de autorizar o prohibir la transformación de su obra, con lo cual solo él podrá decidir quién y bajo qué condiciones realizará esa transformación. De forma que si autoriza la transformación, este acto no dejaría de ser considerado como la cesión o licenciamiento de un derecho patrimonial cualquiera, por el tiempo y las modalidades pactadas, que también conlleva el derecho a participar proporcionalmente de los ingresos que resulten de la explotación de su obra, y para este caso concreto, de la explotación de su obra dentro de otra obra derivada ${ }^{16}$.

Si leemos más detenidamente el artículo 21.2 del TRLPI, veremos cómo la afirmación que se ha hecho antes no será el límite de aplicación del derecho de transformación, porque más allá de facultar al autor original para autorizar la transformación de su obra y recibir una remuneración por ello, se establece una facultad de autorizar cualquier uso (reproducción, distribución, comunicación pública o nueva transformación) de los resultados, es decir, autorizar la explotación de las obras derivadas ${ }^{17}$.

El artículo 21.2 del TRLPI amplía las facultades de explotación del autor de una obra original, porque le otorga un derecho de autorizar (el cual seguramente lleva implícita la contraprestación de también prohibir) cualquier forma de explotación de la obra derivada. De forma tal que habrá dos momentos y dos objetos distintos sobre los que el autor de una obra original tendrá derechos exclusivos de autorizar o prohibir: (i) al momento de permitir o no

conexos en el entorno digital, auspiciado por la Organización Mundial de la Propiedad Intelectual (OMPI), la Sociedad General de Autores y Editores (SGAE) de España y el Ministerio de Industria y Comercio de la República del Paraguay, Asunción, Paraguay, 7-11 de noviembre de 2005. Disponible en: http://www.wipo.int/edocs/mdocs/lac/es/ompi_sgae_da_asu_05/ ompi_sgae_da_asu_05_2.pdf

16 En los términos del artículo 46 TRLPI.

17 Acerca de la sobreposición de derechos en la transformación de obras, DANIEL GeRvais, The Derivative Right, or Why Copyright Law Protects Foxes Better than Hedgehogs, 15 Vanderbilt Journal of Entertainment and Technology Law, 785-855 (2014). Disponible en: http://www. jetlaw.org/wp-content/uploads/2013/05/Gervais.pdf 
la transformación de su obra, para que esta primera derive en otra nueva; y (ii) durante todo el plazo de protección de la obra original para permitir o no la distribución, comunicación pública o nuevas transformaciones (siendo esta una lista abierta) del resultado de la transformación autorizada (de la obra derivada).

Esta interpretación de la ley de propiedad intelectual no es ni mucho menos pacífica. Encontraremos que con este entendimiento se enfrentarán sobre un mismo objeto los derechos exclusivos de los titulares originarios, caso grave y notorio cuando la obra original es parte esencial de la derivada, pero que también preocupa al momento de analizar los casos de obras originales que simplemente inspiraron la derivada o que su incorporación a la segunda es meramente accesoria, ¿tendrá el autor de la obra originaria derechos exclusivos sobre la derivada en este último supuesto también? ¿Deberán funcionar ahora todas las obras derivadas como obras en colaboración o colectivas?

Parece que al entender de forma tan amplia el derecho de transformación sobre la obra original, vamos en contra de preceptos básicos de la misma ley de propiedad intelectual que de forma general otorga todos los derechos al autor de la obra "final" por el mero hecho de la creación, así esta última sea derivada ${ }^{18}$; de allí que el derecho de transformación, entendido de forma "tradicional"19 llegara hasta autorizar o prohibir las modificaciones o adaptaciones y que no transitara hasta el resultado de estas cuando ya existe otro autor originario.

Se podría decir que una redacción de este tipo obedece a la innegable relación que hay entre el derecho de transformación y el derecho moral del autor para hacer respetar la integridad de su obra. Cualquier transformación de una obra - como una traducción, adaptación o en general cualquier modificación - necesariamente implica una alteración de esta y cada autor en cada caso particular podrá entenderla como un atentado a la integridad de su obra. La equiparación entre el derecho de explotación o patrimonial de transformación y el moral de modificación o integridad, aunque tiene

18 Artículo 21.2, primer apartado.

19 Carlos Rogel-VIde, Modificaciones de las obras cinematográficas en la televisión y derechos de los creadores, en Anuario de Propiedad Intelectual 2007, 437-454, Reus, Madrid (2008). 
una distinción práctica bastante sencilla ${ }^{20}$, la confusión conceptual que subyace no es ajena a los ordenamientos jurídicos del derecho civil. En este sentido, encontramos que el verbo "modificar" es utilizado indistintamente para referirse a los derechos morales y a los derechos de explotación o patrimoniales; por ejemplo, el mismo artículo 21 del TRLPI entre los actos que describen la transformación, incluye "cualquier otra modificación"21.

Pero las modificaciones a una obra, aunque estén muy ligadas al derecho moral del autor, hacen parte del mercado de la propiedad intelectual que exige un tipo de gestión más eficiente del que pueden brindar los derechos morales, de allí que sea necesaria la existencia de un derecho de explotación que trate sobre aquellos usos, que también son modificatorios de la obra, pero que no pueden estar limitados dentro del ámbito personalísimo del autor. Es decir, las traducciones o adaptaciones de una obra, como modificaciones de la misma, deben ser derechos de libre disposición por parte del autor y que garanticen el uso o explotación pacíficos por parte del empresario o usuarios en general; entendido que este último se verá seriamente comprometido con la interpretación expuesta del artículo 21.2 del TRLPI.

Esa seguridad jurídica necesaria para explotar las obras en cuanto a los derechos cedidos, como hemos visto, estará en peligro cuando el autor de la obra original pueda en cualquier momento decidir sobre las formas de explotación de la obra derivada que ya autorizó. Como consecuencia, y con esta redacción del artículo 21.2, quien pretenda obtener la autorización de un autor para crear una obra derivada tendrá que especificar contractualmente que se le han cedido, además del derecho de transformación y los que sean necesarios para la explotación de su obra derivada, aquellos que pudiera tener el autor de la obra original sobre la obra derivada o resultado de la transformación.

20 La modificación es toda aquella transformación no autorizada por el autor y que atenta contra la integridad, entendida esta última como la imagen ideal del autor respecto de su obra.

21 Esta misma confusión aparece en el artículo 23 de la Decisión Andina 351, o en la Sentencia C-871 de 2010, mediante la cual la Corte Constitucional en Colombia analiza las facultades del autor-arquitecto sobre su obra. Comunidad Andina, Decisión Andina 351, de 17 de diciembre de 1993, Régimen común sobre derecho de autor y derechos conexos. Disponible en: http:// www.wipo.int/wipolex/es/details.jsp?id=9445. Corte Constitucional, Sentencia C-871/10, 4 de noviembre de 2010, Magistrado ponente Luis Ernesto Vargas-Silva. Disponible en: http:// www.corteconstitucional.gov.co/relatoria/2010/C-871-10.htm 
De forma que hay una solución (aparente ${ }^{22}$ ) al problema que ha de ser enfrentado en el mercado de las transformaciones de carácter comercial; sin embargo, nos deja unos derechos a favor del autor original que el transformador solo podrá tener por cesión expresa, dejando abierta por lo menos una nueva forma de hacer negocios ${ }^{23}$. Pero más allá de nuevos derechos para el autor o cláusulas a ser implementadas en los contratos de transformación, el problema está en cómo afecta esta redacción del artículo 21.2 del TRLPI al propio sistema de propiedad intelectual.

Primero, tendremos que balancear la coexistencia de autores en una misma obra (la derivada), para lo que hay dos posibilidades: entender este tipo de obras como colectivas o en colaboración; y será necesario tomar una decisión al respecto ya que el autor original no es un simple titular de derechos sobre la obra derivada, lo encontramos como un autor que ostenta derechos sobre la misma (más adelante, hablaremos del vínculo entre este autor y la obra derivada). En algunos casos, el autor transformador será quien coordina, edita y divulga la obra derivada, siendo adecuado el estatuto de las obras colectivas ${ }^{24}$; pero no siempre será así, de forma que quedaría abierta la posibilidad de aplicar las reglas de la obra en colaboración ${ }^{25}$. Sin embargo, en este punto, cabe preguntarse si el autor original puede ser considerado como coautor, al mismo tiempo, de la obra derivada. En caso de responder afirmativa o negativamente, el régimen de las obras colectivas se podrá aplicar, debido a que será considerado como "editor" o "productor" y no tanto como un autor. Pero si no realiza este tipo de funciones, el régimen de las obras en colaboración mantiene vigente la pregunta: ¿puede ser considerado el autor original coautor de las obras derivadas de la suya realizada por otros autores?

22 Digo aparente, en la medida en que la cesión de una obra futura traerá sus complicaciones sobre las formalidades que esta cesión deberá cumplir.

23 Que en mi opinión traerá consigo inseguridad jurídica, ante todo en los casos de los contratos de traducción o adaptación cinematográfica ya suscritos a la fecha y sobre los cuales no se ha previsto una cesión en tal sentido.

24 "Definida, pues, por estar integrada por aportaciones de diferentes autores, ninguno de los cuales ostenta un derecho sobre el conjunto de todas ellas". Hermenegildo BAylos-CorrozA, Tratado de Derecho Industrial, 788, $3^{\mathrm{a}}$ ed., Civitas, Navarra (2009).

25 "En ella, la nota esencial se resume en el hecho de que las aportaciones de los diversos autores intervinientes resulte en una obra única". Hermenegildo BAYLos-Corroza, Tratado de Derecho Industrial, 789, $3^{\mathrm{a}}$ ed., Civitas, Navarra (2009). 
A pesar de lo anterior, si el autor transformador, sus agentes o representantes han procurado la cesión o el licenciamiento de los derechos necesarios para poder realizar su actividad transformadora, tal situación parece demostrar la decisión irresoluta de este autor para asumir el riesgo en el proceso creativo de su obra. En este sentido, nos encontramos más cercanos a considerar la relación entre autor transformador y autor original como equivalente a la de las obras colectivas, en la cual el productor (en este caso representado como el autor transformador) es quien tiene la iniciativa del proceso creativo.

De otra parte, nos preguntamos qué derechos se le otorgan al autor de la obra original, ¿son solo derechos de explotación o también tendrá derechos morales? Pues analizando únicamente lo establecido en el artículo 21.2, tenemos que los nuevos derechos son, en principio, patrimoniales: reproducción, distribución, comunicación pública o nueva transformación, lo que tiene sentido si pensamos que el artículo se encaja entre las facultades patrimoniales del autor descritas en el TRLPI; pero si vamos más allá, si observamos la sombra de estos nuevos derechos, nos encontramos con que aquella separación de la que hablamos entre facultades morales (integridad de la obra) y patrimoniales (transformación de la obra) es puesta en duda con el artículo 21.2, ya que está soportando un vínculo personal $^{26}$, no real, entre el autor original y la obra derivada ${ }^{27}$. Por

26 Como lo ha manifestado Justin Hughes: "Se ha observado que los derechos de propiedad son utilizados como medios para proteger los intereses personales o 'personalidad' de los individuos; esto es especialmente cierto en el caso de los derechos de propiedad intelectual que son otorgados sobre las creaciones del ingenio humano. De acuerdo a esto, los defensores de la 'personalidad' se encontrarán en la vanguardia de los 'derechos morales' a favor de los autores" (traducción libre del autor). Justin Hughes, The Personality Interest of Artist and Inventors in Intellectual Property, 16 Cardozo Arts \& Entertainment Law Journal, 81, 76-116 (1998). Disponible en: http://lib.znate.ru/docs/index-69619.html

27 En Estados Unidos, los casos Suntrust v. Houghton Mifflin, Co. (N.D. Ga. 2001) y Salinger v. Colting (S.D.N.Y. 2009), "han demostrado que los titulares de derechos de autor entendieron al derecho de transformación como algo más que un derecho patrimonial, como algo más cercano al derecho moral de integridad" (traducción libre del autor). Deidré A. Keller, Recognizing the Derivative Works Right as a Moral Right: A Case Comparison and Proposal, 63 Case Western Reserve Law Review, 2, 511-553, 548 (2012). Disponible en: http://law.case. edu/journals/LawReview/Documents/63CaseWResLRev2.5.Article.Keller.pdf. United STATES Court of Appeals, Eleventh Circuit, Suntrust Bank v. Houghton Mifflin Company, n. ${ }^{\circ} 01-$ 12200 (N.D. Ga. 2001), decided October 10, 2001. Disponible en: http://caselaw.findlaw.com/ us-11th-circuit/1332488.html. United States Court of Appeals, Second Circuit, Salinger v. Colting (S.D.N.Y. 2009), docket n. ${ }^{\circ}$ 09-2878-cv, decided April 30, 2010. Disponible en: http:// caselaw.findlaw.com/us-2nd-circuit/1521467.html. También Michelle Chatelain, Harry Potter and Prisoner of Copyright Law: Fan Fiction, Derivative Works, and the Fair Use Doctrine, 15 
este motivo se puede poner en duda el carácter exclusivamente patrimonial de la disposición del citado artículo, lo cual genera dudas sobre si se podrá disponer de él contractualmente (como se mencionó antes). Así como Deidré A. Keller lo ha manifestado: "los titulares de derechos de propiedad intelectual alegando infracciones causadas por obras derivadas y los jueces que protegen tales derechos, normalmente están buscando reivindicar un derecho moral en vez de uno patrimonial" 28 .

Una persona no podría tener derechos sobre un bien de propiedad intelectual sin justificación alguna, como la creación o la transmisión (inter vivos o mortis causa); para el caso que estudiamos ¿cuál sería la justificación para otorgar derechos al autor original sobre la obra derivada, al nivelarlo como coautor junto al autor transformador? Si autor es la persona que crea una obra protegida por la propiedad intelectual y en otros casos, el productor será quien edita o divulga bajo su nombre una obra ¿qué papel juega el autor original en la obra derivada que no ha encargado de forma alguna?

Pues bien, estas preguntas podrían llevar a concluir que cuando el artículo 21.2 otorga derechos exclusivos al autor original sobre los resultados de la transformación de su obra, se está reconociendo la inspiración como aporte esencial dentro de la obra derivada ${ }^{29}$. Esta conclusión es muy grave si tenemos en cuenta que la ley de propiedad intelectual ya no solo protegerá la forma especial en la que el autor se expresa artísticamente, sino que ampliará su espectro de protección hasta la idea misma. No cabría discutir si esta protección opera solamente cuando la obra original tenga una importancia sustancial dentro de la obra derivada, escasa ella misma de contenido original protegible, ya que el artículo $21.2 \mathrm{se}$ asegura de trasladar los nuevos derechos del autor original hasta cualquier resultado de la transformación, original o no ${ }^{30}$.

Tulane Journal of Technology and Intellectual Property, 199-217 (2012).

28 Traducción libre del autor. Deidré A. Keller, Recognizing the Derivative Works Right as a Moral Right: A Case Comparison and Proposal, 63 Case Western Reserve Law Review, 2, 511553, 514 (2012). Disponible en: http://law.case.edu/journals/LawReview/Documents/63Case WResLRev2.5.Article.Keller.pdf

29 Jorge Ortega-Doménech, Creación humana y autoría intelectual: diálogos en torno a su fundamentación jurídica, en Sujetos del derecho de autor, 13-42, 20, CésAR Iglesias-Rebollo, coord., Reus, Madrid (2007).

30 Sobre los efectos negativos de tal entendimiento, Oren Bracha \& Talha Syed, Beyond the Incentive-Access Paradigm? Product Differentiation \& Copyright Revisited, 92 Texas Law Review, 1841-1920, 1906 (2014). Disponible en: http://www.texaslrev.com/wp-content/uploads/ 
Desde una declaración de mera aspiración personal, entendiendo estos derechos del autor original sobre las obras derivadas creadas por el autor transformador como un límite a los derechos de propiedad intelectual de este último, quisiera otorgarle una interpretación restrictiva $^{31}$ a la disposición del artículo 21.2 del TRLPI, y de esta forma circunscribir sus efectos a los derechos de explotación listados en la referida disposición normativa, sin que se pudiera extender a los derechos morales.

\section{ANTECEDENTES NORMATIVOS DE LA NUEVA TRANSFORMACIÓN}

El derecho de transformación no ha sido tratado de esta manera siempre, el antiguo artículo 21 del TRLPI decía sobre el derecho de transformación:

La transformación de la obra comprende su traducción, adaptación y cualquier otra modificación en su forma de la que se derive una obra diferente.

Los derechos de propiedad intelectual de la obra resultante de la transformación corresponderán al autor de esta última, sin perjuicio de los derechos del autor de la obra preexistente.

La redacción original del Real Decreto Legislativo 1/1996, de 12 de abril, era clara sobre quién ostenta qué derechos en cada obra; así como se ha dicho, el autor de la obra original tendrá el derecho de autorizar o prohibir cualquier transformación, pero una vez autorizada tal forma de explotación, será el autor transformador quien ostentará los derechos de propiedad intelectual sobre la obra resultante.

El cambio en todo el artículo 21 del TRLPI se dio con motivo de la incorporación al derecho español de la Directiva 96/9/CE del Parlamento Europeo y del Consejo del 11 de marzo de 1996, para la protección de las bases de datos ${ }^{32}$. En este nuevo sistema

BrachaSyed-92-7.pdf

31 Sobre la interpretación restrictiva de los límites a la propiedad intelectual, Delia Lipszyc, Derecho de autor y derechos conexos, 220, $2^{\mathrm{a}}$ ed., United Nations Educational, Scientific and Cultural Organization, UNESCO, Buenos Aires (1993).

32 Sobre el proceso de unificación europea respecto de la protección de datos de carácter personal, Miguel Ángel Bouza-López, El derecho sui generis del fabricante de bases de datos, Reus, 
de protección resultaba de suma importancia asegurar que la simple - reordenación de la base de datos fuese considerada como una transformación que requiere la autorización del autor o titular de derechos; así se añadió el actual párrafo segundo del numeral 1.

El punto que nos interesa, el segundo del artículo 21, fue "aclarado" por la ley de incorporación con el fin de acomodar la redacción al artículo 5.e de la citada Directiva, el cual, efectivamente atribuye al autor de una base de datos el derecho exclusivo, entre otros, de realizar o autorizar cualquier reproducción, distribución, comunicación, exhibición o representación al público de los resultados de los actos de traducción, adaptación, reordenación y cualquier otra modificación de su base de datos.

De allí la redacción del actual artículo 21.2 del TRLPI, que atribuye derechos exclusivos al autor de la obra original sobre los resultados de las transformaciones autorizadas a otros autores. Inútil sería debatir la necesidad de reconocerles tales derechos a los autores-fabricantes de bases de datos ${ }^{33}$. Esta es una Directiva de obligatorio cumplimiento e incorporación al derecho interno, poco más se podrá discutir al respecto; pero sí resulta cuestionable ampliar tal entendimiento a todos los tipos de obras protegidas por la propiedad intelectual. Al fin y al cabo, la protección de las bases de datos es sui generis, especial, distinta al derecho de autor; sin embargo, sí es cuestionable que esa protección modifique la naturaleza misma del derecho de transformación de las obras protegidas por el derecho de autor.

Madrid (2001). Parlamento Europeo y Consejo Europeo, Directiva 96/9/CE del 11 de marzo de 1996, sobre la protección jurídica de las bases de datos. Disponible en: http://europa.eu/ legislation_summaries/internal_market/businesses/intellectual_property/126028_es.htm

33 Miguel Ángel Bouza-López, El derecho sui generis del fabricante de bases de datos, 181, Reus, Madrid (2001). 


\section{EL DROIT DE SUITE COMO EL AMBIENTE PROPICIO PARA EL SEGUIMIENTO DEL DERECHO DE AUTOR EN LAS OBRAS DERIVADAS.}

El derecho de participación, seguimiento o droit de suite ${ }^{34}$ es el derecho de los autores de obras artísticas ${ }^{35}$ a percibir una participación en el precio de las ventas sucesivas, que se hagan en diferentes actos dispositivos posteriores a la primera venta. En legislaciones como la francesa ${ }^{36}$, esa participación está limitada a las ventas en subastas públicas, el artículo 24 de TRLPI, por ejemplo, añade el derecho de seguimiento a las ventas sucesivas realizadas en galerías de arte.

Mucho se ha debatido sobre la naturaleza del droit de suite en la doctrina; la gran mayoría de autores reconoce el debate irresoluto sobre la calificación del derecho de seguimiento como un verdadero derecho de autor o un simple derecho remuneratorio ${ }^{37}$. Este debate no abandonará el estadio puramente académico, en la medida en que la regulación de tal derecho se ha encargado de restringirlo a tal punto que cierra los espacios para que su naturaleza tenga una consecuencia práctica; así el artículo 24 del TRLPI lo ha calificado como un derecho irrenunciable y transmisible únicamente mortis causa, es decir, un derecho patrimonial que está en cabeza del autor de una obra artística o plástica.

Sin embargo, un aspecto poco analizado, por lo menos en la doctrina del derecho civil, es la estrecha relación entre el droit de suite y el derecho moral del autor sobre su obra. Es decir, la posi-

34 Más conocido por su denominación en francés, debido a su origen en la ley gala de 1920. DeLIA LIPSZYC, Derecho de autor y derechos conexos, 212, $2^{\mathrm{a}}$ ed., United Nations Educational, Scientific and Cultural Organization, UNESCO, Buenos Aires (1993). Jorge Ortega-DomÉnech, Obra plástica y derechos de autor, 147, Reus, Madrid (2000).

35 Hacemos referencia a obra artística, equiparable con la obra plástica, definida como "[...] obras que se manifiestan por medio de la forma y el color. Se da forma o color a materias preexistentes. De ahí la importancia de las líneas, los planos, las dimensiones, los volúmenes, la intensidad y variedad de los colores y sus tonalidades". Rodrigo Bercovitz RodríGuezCANo, coord., autor, Comentarios a la ley de propiedad intelectual, 228, $3^{\mathrm{a}}$ ed., Tecnos, Madrid (1989/1997).

36 André Lucas, \& Henri Jacques Lucas, Traité de la propiété littéraire \& artistique, 291, Litec, Paris (1994).

37 Incluso algunos lo califican como un derecho conexo al derecho de autor, Matías ValLèsRoDríguez, El derecho de participación de los autores de obras plásticas ("droit de suite") en la nueva ley de propiedad intelectual, 96 Revista General de Legislación y Jurisprudencia, 4, 619-624 (1988). 
bilidad del autor de una obra plástica de "seguir" la suerte que el soporte material alcance en el futuro, así él se haya desprendido de la propiedad dominical de esos materiales, es el reconocimiento de la obra plástica como expresión de la personalidad misma del autor, unida a este último por un cordón umbilical indestructible (irrenunciable) $^{38}$

Ese reconocimiento expreso a la relación entre un derecho patrimonial, como el droit de suite, y el derecho moral del autor nos sirve para comprender la amplitud de facultades consagradas a favor del autor de la obra original en el artículo 21.2 del TRLPI (la relación entre el derecho patrimonial de transformación y el derecho moral de integridad).

Cuando se habla desde el derecho de autor (derecho civil) del droit de suite, y se hace reconocimiento al mito del autor "miserable" y la injusticia de la situación que compara su suerte con la de la obra vendida por muchísimo dinero ${ }^{39}$, se busca equiparar a la persona (autor) con la suerte del soporte material en donde su obra ha sido expresada. En ese mismo sentido, cuando el artículo 21.2 del TRLPI amplía la facultad del autor original a autorizar la explotación de las obras derivadas de otros autores, está identificando el estado (uso) de la expresión artística con los intereses personales del autor. Todo, con una explicación personalísima o moral y con consecuencias patrimoniales.

En este sentido, el droit de suite no constituye el antecedente directo de la ampliación del derecho de transformación, pero sí evidencia el medio ambiente en el que un derecho tan amplio puede subsistir; ese escenario en donde el derecho patrimonial o de explotación se identifica directamente con la persona-autor, y le permite ostentar prerrogativas que exceden la diferenciación de propiedades materiales (droit de suite) e intelectuales (derecho de transformación). En conclusión, el artículo 21.2 del TRLPI le permite al autor original seguir la suerte de su obra y participar de la explotación, incluso cuando la nueva situación de esta deviene de una transformación o modificación hecha por un tercero.

38 Sobre el reconocimiento de esta relación, AleXAnder Bussey, The Incompatibility of Droit de Suite with Common Law Theories of Copyright, 23 Fordham Intellectual Property, Media \& Entertainment Law Journal, 1063-1104, 1076 (2013). Disponible en: http://papers.ssrn.com/ sol3/papers.cfm?abstract_id $=2248177$

39 RAMÓN CASAS-VAlLÈs, La protección de los artistas plásticos en el derecho español, en I Congreso Iberoamericano de Propiedad Intelectual. Derechos de autor y derechos conexos en los umbrales del año 2000, 259-278, tomo I, Ministerio de Cultura, Madrid (1991), citado por Jorge OrtegADoménech, Obra plástica y derechos de autor, 179, Reus, Madrid (2000). 


\section{CONCLUSIONES}

Cuando el artículo 133 del TRLPI define el objeto de protección sui generis de las bases de datos como aquella "inversión sustancial (...) que realiza su fabricante, ya sea de medios financieros, empleo de tiempo, esfuerzo, energía u otros de similar naturaleza, para obtención, verificación o presentación de su contenido", está reconociendo la ausencia de la originalidad en tal objeto de protección. Y precisamente, ante esa carencia de originalidad en un esquema de protección mediante la propiedad inmaterial, surge la posibilidad de establecer la continuidad del derecho del titular, incluso más allá del objeto protegido. Allí no se está protegiendo la expresión original de una idea, se protege la inversión realizada, la cual seguramente se verá afectada si no se le asegura una participación al inversionista en los desarrollos futuros de su inversión.

Esta realidad de las bases de datos no quedó circunscrita a la protección sui generis especialmente diseñada para este objeto de protección; escurrió hasta el derecho de autor con la modificación del artículo 21 del TRLPI, en la que se otorgó expresamente un derecho exclusivo al autor de la obra original para autorizar los usos que el autor transformador haga de la obra derivada de la suya.

Por supuesto, tal relectura del derecho de transformación genera más preguntas que certezas: ¿cómo será la relación entre autor original y autor transformador?, ¿confluirán como en la relación de obra colectiva, o como en la de las obras por colaboración?, ¿tal participación estará limitada a algunos derechos patrimoniales, o será tan amplia como está descrita en el artículo 21.2?

Resulta claro que este cambio del tradicional derecho de transformación puede subsistir perfectamente en el ambiente del derecho de autor (derecho civil), toda vez que para este sistema no resulta extraña la interlocución entre los derechos morales y los patrimoniales en la protección de la persona como autor de una obra. En este caso, veremos cómo el derecho moral a la integridad de la obra se desarrolla en el nuevo derecho patrimonial de transformación, para de esta forma asegurar al autor original 
continuar su derecho en las obras derivadas de la transformación. Lógica utilizada en el sistema del derecho de autor de tradición civil para sustentar el droit de suite o, mejor dicho, derecho de seguimiento del autor, de continuidad del derecho. 


\section{BIBLIOGRAFÍA}

\section{Libros}

Baylos-Corroza, Hermenegildo, Tratado de Derecho Industrial, $3^{a}$ ed., Civitas, Navarra (2009).

Bercovitz Rodríguez-CAno, Rodrigo, coord., autor, Comentarios a la ley de propiedad intelectual, $3^{\mathrm{a}}$ ed., Tecnos, Madrid (1997).

Bercovitz Rodríguez-CAno, Rodrigo, coord., autor, Manual de propiedad intelectual, Tirant Lo Blanch, Valencia (2001).

Bouza-López, Miguel Ángel, El derecho sui generis del fabricante de bases de datos, Reus, Madrid (2001).

LipsZyc, Delia, Derecho de autor y derechos conexos, $2^{\mathrm{a}}$ ed., United Nations Educational, Scientific and Cultural Organization, UNESCO, Buenos Aires (1993).

López-SÁnchez, Cristina, La transformación de la obra intelectual, Dykinson, Madrid (2008).

Lucas, André \& Lucas, Henri Jacques, Traité de la propiété littéraire \& artistique, Litec, Paris (1994).

Ortega-Doménech, Jorge, Obra plástica y derechos de autor, Reus, Madrid (2000).

\section{Contribución en obras colectivas}

Casas-Vallès, Ramón, La protección de los artistas plásticos en el derecho español, en I Congreso Iberoamericano de Propiedad Intelectual. Derechos de autor y derechos conexos en los umbrales del año 2000, 259-278, tomo I, Ministerio de Cultura, Madrid (1991).

OrTega-Doménech, JoRge, Creación humana y autoría intelectual: diálogos en torno a su fundamentación jurídica, en Sujetos del derecho de autor, 13-42, CÉSAR IgLESIASRebollo, coord., Reus, Madrid (2007).

\section{Revistas}

Bracha, Oren \& Syed, Talha, Beyond the Incentive-Access Paradigm? Product Differentiation \& Copyright Revisited, 92 Texas Law Review, 1841-1920 (2014). Disponible en: http://www.texaslrev.com/wp-content/uploads/BrachaSyed-92-7. pdf

Bussey, Alexander, The Incompatibility of Droit de Suite with Common Law Theories of Copyright, 23 Fordham Intellectual Property, Media \& Entertainment Law Journal, 1063-1104 (2013). Disponible en: http://papers.ssrn.com/sol3/papers. cfm?abstract_id=2248177

Chatelain, Michelle, Harry Potter and Prisoner of Copyright Law: Fan Fiction, Derivative Works, and the Fair Use Doctrine, 15 Tulane Journal of Technology 
and Intellectual Property, 199-217 (2012).

Damich, Edward J., The Right of Personality: A Common-Law Basis for the Protection of the Moral Rights of Authors, 23 Georgia Law Review, 1-24 (1988).

Gervais, Daniel, The Derivative Right, or Why Copyright Law Protects Foxes Better than Hedgehogs, 15 Vanderbilt Journal of Entertainment and Technology Law, 785-855 (2014). Disponible en: http://www.jetlaw.org/wp-content/uploads/2013/05/ Gervais.pdf

Hughes, Justin, The Personality Interest of Artist and Inventors in Intellectual Property, 16 Cardozo Arts \& Entertainment Law Journal, 81, 76-116 (1998). Disponible en: http://lib.znate.ru/docs/index-69619.html

Keller, Deidré A., Recognizing the Derivative Works Right as a Moral Right: A Case Comparison and Proposal, 63 Case Western Reserve Law Review, 2, 511-553 (2012). Disponible en: http://law.case.edu/journals/LawReview/Documents/63CaseWR esLRev2.5.Article.Keller.pdf

Landes, William M. \& Posner, Richard A., An Economic Analysis of Copyright Law, 18 Journal of Legal Studies, 2, 325-363 (1989). Disponible en: http://www.sfu. ca/ allen/copyright.pdf

Lunney, Glynn S., JR., Copyright, Derivative Works, and the Economics of Complements, 12 Vanderbilt Journal of Entertainment and Technology Law, 4, 779-817 (2010). Disponible en: http://www.jetlaw.org/wp-content/uploads/2011/09/Lunney_ online.pdf

Rogel-Vide, Carlos, Modificaciones de las obras cinematográficas en la televisión y derechos de los creadores, en Anuario de Propiedad Intelectual 2007, 437-454, Reus, Madrid (2008).

Samuelson, Pamela, The Quest for a Sound Conception of Copyright's Derivate Work Right, 101 Georgetown Law Journal, 1505-1564 (2013). Disponible en: http:// georgetownlawjournal.org/files/2013/09/Samuelson.pdf

Vallès-Rodríguez, Matías, El derecho de participación de los autores de obras plásticas ("droit de suite") en la nueva ley de propiedad intelectual, 96 Revista General de Legislación y Jurisprudencia, 4, 619-624 (1988).

\section{Documentos, informes, reportes}

Antequera, RicARdo, Las limitaciones y excepciones al derecho de autor y los derechos conexos en el entorno digital. XI Curso académico regional OMPI/SGAE sobre derecho de autor y derechos conexos para países de América Latina: el derecho de autor y los derechos conexos en el entorno digital, auspiciado por la Organización Mundial de la Propiedad Intelectual (OMPI), la Sociedad General de Autores y Editores (SGAE) de España y el Ministerio de Industria y Comercio de la República del Paraguay, Asunción, Paraguay, 7-11 de noviembre de 2005. Disponible en: http://www.wipo.int/edocs/mdocs/lac/es/ompi_sgae_da_asu_05/ ompi_sgae_da_asu_05_2.pdf 


\section{Normatividad española}

España, Ley 1/2000, de 7 de enero, de Enjuiciamiento Civil, 7 Boletín Oficial del Estado, $B O E, 8$ de enero de 2000. Disponible en: https://www.boe.es/buscar/act. php?id=BOE-A-2000-323

España, Ley 21/2014, de 4 de noviembre, por la que se modifica el Texto Refundido de la Ley de Propiedad Intelectual, aprobado por Real Decreto Legislativo 1/1996, de 12 de abril, y la Ley 1/2000, de 7 de enero, de Enjuiciamiento Civil, 268 Boletín Oficial del Estado, BOE, 5 de noviembre de 2014. Disponible en: https://www. boe.es/diario_boe/txt.php?id=BOE-A-2014-11404

España, Real Decreto Legislativo 1/1996, de 12 de abril, por el que se aprueba el texto refundido de la Ley de Propiedad Intelectual, regularizando, aclarando y armonizando las disposiciones legales vigentes sobre la materia, 97 Boletín Oficial del Estado, BOE, 22 de abril de 1996. Disponible en: https://www.boe.es/ diario_boe/txt.php?id=BOE-A-1996-8930

Parlamento Europeo y Consejo Europeo, Directiva 96/9/CE del 11 de marzo de 1996, sobre la protección jurídica de las bases de datos. Disponible en: http://europa. eu/legislation_summaries/internal_market/businesses/intellectual_property/ 126028_es.htm

\section{Jurisprudencia internacional}

Comunidad Andina, Decisión Andina 351, de 17 de diciembre de 1993, Régimen común sobre derecho de autor y derechos conexos. Disponible en: http://www.wipo.int/ wipolex/es/details.jsp?id=9445

\section{Jurisprudencia colombiana}

Corte Constitucional, Sentencia C-871/10, 4 de noviembre de 2010, Magistrado ponente Luis Ernesto Vargas-Silva. Disponible en: http://www.corteconstitucional.gov. co/relatoria/2010/C-871-10.htm

\section{Casos}

United States Court of Appeals, Eleventh Circuit, Suntrust Bank v. Houghton Mifflin Company, n. ${ }^{\circ}$ 01-12200 (N.D. Ga. 2001), decided October 10, 2001. Disponible en: http://caselaw.findlaw.com/us-11th-circuit/1332488.html

United States Court of Appeals, Second Circuit, Salinger v. Colting (S.D.N.Y. 2009), docket n. ${ }^{\circ}$ 09-2878-cv, decided April 30, 2010. Disponible en: http://caselaw. findlaw.com/us-2nd-circuit/1521467.html 